\title{
ON TWO METHODS FOR RECONSTRUCTING HOMOGENEOUS HYPERSURFACE SINGULARITIES FROM THEIR MILNOR ALGEBRAS*
}

\author{
A. V. ISAEV ${ }^{\dagger}$
}

\begin{abstract}
By the well-known Mather-Yau theorem, a complex hypersurface germ $\mathcal{V}$ with isolated singularity is fully determined by its moduli algebra $\mathcal{A}(\mathcal{V})$. The proof of this theorem does not provide an explicit procedure for recovering $\mathcal{V}$ from $\mathcal{A}(\mathcal{V})$, and finding such a procedure is a long-standing open problem. In the present paper we survey and compare two recently proposed methods for reconstructing $\mathcal{V}$ from $\mathcal{A}(\mathcal{V})$ up to biholomorphic equivalence under the assumption that the singularity of $\mathcal{V}$ is homogeneous (in which case $\mathcal{A}(\mathcal{V})$ coincides with the Milnor algebra of $\mathcal{V}$ ). As part of our discussion of one of the methods, we give a characterization of the algebras arising from finite polynomial maps with homogeneous components of equal degrees.
\end{abstract}

Key words. Isolated hypersurface singularities, Milnor algebras, the Mather-Yau theorem.

AMS subject classifications. 32S25, $13 \mathrm{H} 10$.

1. Introduction. Let $\mathcal{O}_{n}$ be the local complex algebra of holomorphic function germs at the origin in $\mathbb{C}^{n}$ with $n \geq 2$. For a hypersurface germ $\mathcal{V}$ at the origin (considered with its reduced complex structure) denote by $I(\mathcal{V})$ the ideal of elements of $\mathcal{O}_{n}$ that vanish on $\mathcal{V}$. Fix a generator $f$ of $I(\mathcal{V})$ and let $\mathcal{A}(\mathcal{V})$ be the quotient of $\mathcal{O}_{n}$ by the ideal generated by $f$ and all its first-order partial derivatives. The algebra $\mathcal{A}(\mathcal{V})$, called the moduli algebra or Tjurina algebra of $\mathcal{V}$, is in fact independent of the choice of $f$ as well as the coordinate system near the origin. Furthermore, the moduli algebras of biholomorphically equivalent hypersurface germs are isomorphic. It is clear that $\mathcal{A}(\mathcal{V})$ is non-zero if and only if $\mathcal{V}$ is singular. We assume that $0<\operatorname{dim}_{\mathbb{C}} \mathcal{A}(\mathcal{V})<\infty$, which occurs if and only if the singularity of $\mathcal{V}$ is isolated (see, e.g. Chapter 1 in [GLS]).

By the well-known Mather-Yau theorem (see $[\mathrm{MY}]$ ), two hypersurface germs $\mathcal{V}_{1}$, $\mathcal{V}_{2}$ in $\mathbb{C}^{n}$ with isolated singularities are biholomorphically equivalent if their moduli algebras $\mathcal{A}\left(\mathcal{V}_{1}\right), \mathcal{A}\left(\mathcal{V}_{2}\right)$ are isomorphic. Thus, given the dimension $n$, the moduli algebra $\mathcal{A}(\mathcal{V})$ determines $\mathcal{V}$ up to biholomorphism. For example, if $\operatorname{dim}_{\mathbb{C}} \mathcal{A}(\mathcal{V})=1$, then $\mathcal{V}$ is biholomorphic to the germ of the hypersurface $\left\{z_{1}^{2}+\cdots+z_{n}^{2}=0\right\}$, and if $\operatorname{dim}_{\mathbb{C}} \mathcal{A}(\mathcal{V})=2$, then $\mathcal{V}$ is biholomorphic to the germ of the hypersurface $\left\{z_{1}^{2}+\cdots+\right.$ $\left.z_{n-1}^{2}+z_{n}^{3}=0\right\}$. The proof of the Mather-Yau theorem does not provide an explicit procedure for recovering the germ $\mathcal{V}$ from the algebra $\mathcal{A}(\mathcal{V})$ in general, and finding a way for reconstructing $\mathcal{V}$ (or at least some invariants of $\mathcal{V}$ ) from $\mathcal{A}(\mathcal{V})$ is an interesting open question, called the reconstruction problem (cf. [Y1], [Y2], [Sch]). Recently, we have proposed two methods for restoring $\mathcal{V}$ from $\mathcal{A}(\mathcal{V})$ up to biholomorphic equivalence under the assumption that the singularity of $\mathcal{V}$ is homogeneous (see [EI], [I2], [IK]). In the present paper we survey and compare these two methods.

Let $\mathcal{V}$ be a hypersurface germ having an isolated singularity. The singularity of $\mathcal{V}$ is said to be homogeneous if for some generator $f$ of $I(\mathcal{V})$ there is a coordinate system $z_{1}, \ldots, z_{n}$ near the origin in which $f$ becomes the germ of a homogeneous polynomial $Q$. It then follows that this statement holds for any generator of $I(\mathcal{V})$. Indeed, if $g$ is another generator of $I(\mathcal{V})$, then $g=u f$, where $u$ is a unit in $\mathcal{O}_{n}$. It is now easy to check

\footnotetext{
*Received August 5, 2012; accepted for publication February 25, 2013.

$\dagger$ Department of Mathematics, The Australian National University, Canberra, ACT 0200, Australia (alexander.isaev@anu.edu.au).
} 
that $g$ becomes the germ of $Q$ in the coordinates $\left[U\left(z_{1}, \ldots, z_{n}\right)\right]^{1 / \operatorname{deg} Q} \cdot\left(z_{1}, \ldots, z_{n}\right)$, where $U$ is a function representing $u$.

If the singularity of $\mathcal{V}$ is homogeneous, then any generator $f$ of $I(\mathcal{V})$ lies in the Jacobian ideal $\mathcal{J}(f)$ in $\mathcal{O}_{n}$, which is the ideal generated by all first-order partial derivatives of $f$ with respect to some coordinate system. Hence, for a homogeneous singularity, $\mathcal{A}(\mathcal{V})$ coincides with the Milnor algebra $\mathcal{O}_{n} / \mathcal{J}(f)$ for any $f$. Therefore, in this case the algebra $\mathcal{A}(\mathcal{V})$ is a complete intersection, which implies that $\mathcal{A}(\mathcal{V})$ is Gorenstein (see $[\mathrm{B}]$ ). Recall that a local complex commutative associative algebra $\mathcal{A}$ with $1<\operatorname{dim}_{\mathbb{C}} \mathcal{A}<\infty$ is a Gorenstein ring if and only if for the annihilator $\operatorname{Ann}(\mathfrak{m}):=\{x \in \mathfrak{m}: x \mathfrak{m}=0\}$ of its maximal ideal $\mathfrak{m}$ one has $\operatorname{dim}_{\mathbb{C}} \operatorname{Ann}(\mathfrak{m})=1$ (see, e.g. $[\mathrm{H}])$. Furthermore, if the singularity of $\mathcal{V}$ is homogeneous, the algebra $\mathcal{A}(\mathcal{V})$ is (non-negatively) graded, i.e. it can be represented as $\mathcal{A}(\mathcal{V})=\oplus_{i \geq 0} \mathcal{L}_{i}$, where $\mathcal{L}_{i}$ are linear subspaces of $\mathcal{A}(\mathcal{V})$ with $\mathcal{L}_{0} \simeq \mathbb{C}$ and $\mathcal{L}_{i} \mathcal{L}_{j} \subset \mathcal{L}_{i+j}$ for all $i, j$.

Now, we let $Q(z)$, with $z:=\left(z_{1}, \ldots, z_{n}\right)$, be a holomorphic $(m+1)$-form on $\mathbb{C}^{n}$, i.e. a homogeneous polynomial of degree $m+1$ in the variables $z_{1}, \ldots, z_{n}$, where $m \geq 2$. Consider the germ $\mathcal{V}_{Q}$ of the hypersurface $\{Q(z)=0\}$ and assume that: (i) the singularity of $\mathcal{V}_{Q}$ is isolated, and (ii) the germ of $Q$ generates $I\left(\mathcal{V}_{Q}\right)$. These two conditions are equivalent to the non-vanishing of the discriminant $\Delta(Q)$ of $Q$ (see Chapter 13 in [GKZ]). Both methods discussed in this paper aim at recovering the form $Q$ (hence the germ $\mathcal{V}_{Q}$ ) from the algebra $\mathcal{A}\left(\mathcal{V}_{Q}\right)$ up to linear equivalence, where two forms $Q_{1}, Q_{2}$ on $\mathbb{C}^{n}$ are called linearly equivalent if there exists a non-degenerate linear transformation $L$ of $\mathbb{C}^{n}$ such that $Q_{2}=Q_{1} \circ L$.

First, in Section 2 we review the method introduced in [IK]. Define $\mathbf{Q}$ to be the gradient map $\mathbf{Q}: \mathbb{C}^{n} \rightarrow \mathbb{C}^{n}, z \mapsto \operatorname{grad} Q(z)$. The condition $\Delta(Q) \neq 0$ means that the fiber $\mathbf{Q}^{-1}(0)$ consists of 0 alone, i.e. $\mathbf{Q}$ is finite at the origin. The main content of this method is recovering the map $\mathbf{Q}$ from $\mathcal{A}\left(\mathcal{V}_{Q}\right)$ up to linear equivalence, where we say that two maps $\Phi_{1}, \Phi_{2}: \mathbb{C}^{n} \rightarrow \mathbb{C}^{n}$ are linearly equivalent if there exist non-degenerate linear transformations $L_{1}, L_{2}$ of $\mathbb{C}^{n}$ such that $\Phi_{2}=L_{1} \circ \Phi_{1} \circ L_{2}$. In fact, in [IK] we consider a more general situation. Let $p_{1}, \ldots, p_{n}$ be holomorphic $m$-forms on $\mathbb{C}^{n}$ and $I$ the ideal in $\mathcal{O}_{n}$ generated by the germs of these forms at the origin. Define $\mathbf{P}$ to be the map of $\mathbb{C}^{n}$ given by $z \mapsto\left(p_{1}(z), \ldots, p_{n}(z)\right)$ and set $\mathcal{A}_{\mathbf{P}}:=\mathcal{O}_{n} / I$. We assume that $\operatorname{dim}_{\mathbb{C}} \mathcal{A}_{\mathbf{P}}<\infty$, which occurs if and only if $\mathbf{P}$ is finite at the origin, i.e. $\mathbf{P}^{-1}(0)=\{0\}$ (see Chapter 1 in [GLS]). The latter condition is equivalent to the non-vanishing of the resultant of $p_{1}, \ldots, p_{n}$ (see Chapter 13 in [GKZ]). In [IK] we proposed a procedure (which requires only linear-algebraic manipulations) for explicitly recovering the map $\mathbf{P}$ from $\mathcal{A}_{\mathbf{P}}$ up to linear equivalence. Applying this procedure to the algebra $\mathcal{A}\left(\mathcal{V}_{Q}\right)$ arising from $Q$, one obtains a map $\mathbf{Q}^{\prime}$ linearly equivalent to $\mathbf{Q}$. It is then not hard to derive from $\mathbf{Q}^{\prime}$ an $(m+1)$-form $Q^{\prime}$ linearly equivalent to $Q$. To demonstrate how the above method works, in [IK] we applied it to the family of simple elliptic singularities of type $\widetilde{E}_{6}$. A sketch of this example is included in Section 2.

A priori, one can attempt to apply the procedure of [IK] to any complex commutative associative finite-dimensional algebra. Therefore, a natural problem is to characterize the algebras isomorphic to $\mathcal{A}_{\mathbf{P}}$ for some $\mathbf{P}$ as above. In the case of gradient maps, this characterization problem is a special case of the well-known recognition problem for the moduli algebras of general isolated hypersurface singularities and the corresponding Lie algebras of derivations (see, e.g. [Y1], [Y2], [Sch]). In Section 2 we give an elementary solution of the recognition problem for the algebras $\mathcal{A}_{\mathbf{P}}$ prior to our review of the reconstruction method of [IK].

Further, in Section 3 we discuss the method proposed in [EI] (see also [I2]). As 
will be explained below, this method depends on a conjecture that has only been confirmed for a number of special cases but is open in full generality. In fact, in [EI] we construct certain numerical invariants of complex graded Gorenstein algebras of finite vector space dimension greater than 2. Our construction is based on a criterion for two such algebras to be isomorphic given in article [FIKK] (see also [FK], [I1], [I3] for results on algebras over arbitrary fields of characteristic zero). The criterion is stated in terms of certain polynomials. Indeed, as explained in Section 3, to every complex Gorenstein algebra $\mathcal{A}$ with $2<\operatorname{dim}_{\mathbb{C}} \mathcal{A}<\infty$ one can associate polynomials of a special form on $\mathfrak{n}:=\mathfrak{m} / \operatorname{Ann}(\mathfrak{m})=\mathfrak{m} / \mathfrak{m}^{\nu}$, called nil-polynomials, of degree $\nu$ with vanishing constant and linear terms, where $\nu \geq 2$ is the nil-index of $\mathfrak{m}$. In [FIKK] we showed that two complex graded Gorenstein algebras $\mathcal{A}_{1}, \mathcal{A}_{2}$ are isomorphic if and only if some (hence any) nil-polynomials $P_{1}, P_{2}$ arising from $\mathcal{A}_{1}, \mathcal{A}_{2}$, respectively, are linearly equivalent, that is, there exist $c \in \mathbb{C}^{*}$ and a linear isomorphism $L: \mathfrak{n}_{1} \rightarrow \mathfrak{n}_{2}$ such that $c P_{1}=P_{2} \circ L$. The homogeneous component $P^{[s]}$ of degree $s$ of any nilpolynomial $P$ is in fact a polynomial on $\mathfrak{n} / \mathfrak{n}^{\nu+2-s} \simeq \mathfrak{m} / \mathfrak{m}^{\nu+2-s}$ for $s=2, \ldots, \nu$. It then follows that for every $s$ and for any absolute classical invariant $\mathcal{I}$ of forms of degree $s$ on $\mathfrak{m} / \mathfrak{m}^{\nu+2-s}$, the value $\mathcal{I}\left(P^{[s]}\right)$ is invariantly defined. Thus, application of absolute classical invariants to homogeneous components of nil-polynomials yields numerical invariants of complex graded Gorenstein algebras (see Theorem 3.2).

For a hypersurface germ $\mathcal{V}$ having a homogeneous singularity, Theorem 3.2 provides a way for extracting a collection of invariants of $\mathcal{V}$ from its moduli algebra $\mathcal{A}(\mathcal{V})$ if $\operatorname{dim}_{\mathbb{C}} \mathcal{A}(\mathcal{V})>2$, and these invariants arise from absolute classical invariants of forms of degree $s$ on $\mathfrak{m}(\mathcal{V}) / \mathfrak{m}(\mathcal{V})^{\nu+2-s}$ for $s=2, \ldots, \nu$, where $\mathfrak{m}(\mathcal{V})$ is the maximal ideal of $\mathcal{A}(\mathcal{V})$. In $[\mathrm{EI}]$ we proposed a conjecture, which states that the form $Q$ is determined, up to linear equivalence, by the invariants supplied by Theorem 3.2 just for the case $s=\nu$ (see Conjecture 3.4 below). Namely, the conjecture asserts that one can recover the values of all the absolute invariants for the form $Q$ from absolute invariants of forms of degree $\nu$ on $\mathfrak{m}\left(\mathcal{V}_{Q}\right) / \mathfrak{m}\left(\mathcal{V}_{Q}\right)^{2}$ by evaluating the latter for the corresponding polynomials $P^{[\nu]}$. On the other hand, the linear equivalence problem for forms with non-vanishing discriminant is solved by absolute classical invariants of a special form (see formula (3.1)).

Observe now that $\operatorname{dim}_{\mathbb{C}} \mathfrak{m}\left(\mathcal{V}_{Q}\right) / \mathfrak{m}\left(\mathcal{V}_{Q}\right)^{2}=n$. Also, by a well-known result in [Sa] one has $\nu=n(m-1)$. Thus, starting with any form $Q$ on $\mathbb{C}^{n}$ of degree $m+1$ satisfying the condition $\Delta(Q) \neq 0$, upon identification of $\mathfrak{m}\left(\mathcal{V}_{Q}\right) / \mathfrak{m}\left(\mathcal{V}_{Q}\right)^{2}$ with $\mathbb{C}^{n}$, from any nil-polynomial $P$ on $\mathfrak{m}\left(\mathcal{V}_{Q}\right) / \mathfrak{m}\left(\mathcal{V}_{Q}\right)^{\nu}$ one produces another form $P^{[\nu]}$ on $\mathbb{C}^{n}$ of degree $n(m-1)$, and we say that $P^{[\nu]}$ is associated to $Q$ (any two associated forms coincide up to scale). We note that such forms were considered in [Ea] without introducing nil-polynomials (cf. [Em]). In this terminology, one interesting consequence of Conjecture 3.4 is that the invariant theory of forms of a fixed degree with non-vanishing discriminant is fully described by that of their associated forms. Calculations done in [Ea] show that the conjecture holds for binary quartics $(n=2, m=3)$ and ternary cubics $(n=3, m=2)$. Furthermore, in [EI] the conjecture was confirmed for binary quintics $(n=2, m=4)$ and binary sextics $(n=2, m=5)$.

Our second method for recovering $Q$ from $\mathcal{A}\left(\mathcal{V}_{Q}\right)$ is based on Conjecture 3.4. It is not as explicit as the first one even if the conjecture is assumed to hold. Indeed, while computing a form $\widetilde{Q}$ associated to $Q$ is easy, in order to reconstruct $Q$ from $\widetilde{Q}$ up to linear equivalence one would also need to: (a) find (finitely many) generators of the algebra of all absolute invariants given by formula (3.1), (b) establish how exactly these generators are related to absolute invari- 
ants of forms of degree $n(m-1)$, and (c) solve the following "fitting problem": find a form of degree $m+1$ with non-vanishing discriminant on $\mathbb{C}^{n}$ for which the generators take prescribed values (see (3.3) for details). Tasks (a)-(c) are rather non-trivial and make our second method less attractive in comparison to the first one. On the other hand, finding $\widetilde{Q}$ is much more straightforward than performing the computations required by the first method (see (3.4)). Therefore, if one comes up with a procedure of extracting $Q$ from $\widetilde{Q}$ in an easier way, the second method may become quite useful as well. Analogously to Section 2, we conclude Section 3 by an example of application of the second method to simple elliptic singularities of type $\widetilde{E}_{6}$, in which case tasks (a)-(c) are well-understood.

Acknowledgement. This work is supported by the Australian Research Council.

2. The first method. For fixed $m, n \geq 2$ let $\mathbf{P}: \mathbb{C}^{n} \rightarrow \mathbb{C}^{n}$ be a map that is finite at the origin and whose components $p_{1}, \ldots, p_{n}$ are holomorphic $m$-forms on $\mathbb{C}^{n}$. Define $I$ to be the ideal in $\mathcal{O}_{n}$ generated by the germs of these forms at the origin and $\mathcal{A}_{\mathbf{P}}:=\mathcal{O}_{n} / I$. Since $m \geq 2$, one has $\operatorname{dim}_{\mathbb{C}} \mathcal{A}_{\mathbf{P}} \geq n+1>1$. Before reviewing the method of $[\mathrm{IK}]$ for recovering the map $\mathbf{P}$ from $\mathcal{A}_{\mathbf{P}}$, we will give a simple characterization of the algebras that arise from finite homogeneous polynomial maps as above among all complex commutative associative finite-dimensional algebras.

Let $\mathcal{A}$ be a local unital complex commutative associative algebra with $1<$ $\operatorname{dim}_{\mathbb{C}} \mathcal{A}<\infty$. We say that $\mathcal{A}$ has Property $(*)$ if it satisfies conditions $(*)_{1}-(*)_{3}$ stated below. In what follows $\mathfrak{m}$ is the (unique) maximal ideal of $\mathcal{A}$ and $\operatorname{Gr}(\mathcal{A})$ the associated graded algebra of $\mathcal{A}$ :

$$
\operatorname{Gr}(\mathcal{A}):=\bigoplus_{i=0}^{\nu} \mathfrak{m}^{i} / \mathfrak{m}^{i+1},
$$

where $\mathfrak{m}^{0}:=\mathcal{A}$, with the product operation defined in the obvious way. Here $\nu$ is the nil-index of $\mathfrak{m}$, i.e. the largest integer $\mu$ with $\mathfrak{m}^{\mu} \neq 0$ (note that $\mathfrak{m}$ is a nilpotent algebra by Nakayama's lemma).

$(*)_{1}: \mathcal{A}$ is a standard graded algebra, i.e. it can be represented in the form

$$
\mathcal{A}=\bigoplus_{i \geq 0} \mathcal{L}_{i}
$$

where $\mathcal{L}_{i}$ are linear subspaces of $\mathcal{A}$ with $\mathcal{L}_{0} \simeq \mathbb{C}, \mathcal{L}_{i} \mathcal{L}_{j} \subset \mathcal{L}_{i+j}$ for all $i, j$, and $\mathcal{L}_{l}=\mathcal{L}_{1}^{l}$ for all $l \geq 1$. Notice that

$$
\mathfrak{m}=\bigoplus_{i=1}^{\nu} \mathcal{L}_{i}
$$

$\mathcal{L}_{i}=0$ for $i>\nu$, and $\mathcal{L}_{i}$ is a complement to $\mathfrak{m}^{i+1}$ in $\mathfrak{m}^{i}$ for all $i \geq 0$. Hence $\mathcal{A}$ is a standard graded algebra if and only if $\mathcal{A}$ is isomorphic to $\operatorname{Gr}(\mathcal{A})$.

$(*)_{2}: \mathcal{A}$ is a complete intersection.

$(*)_{3}$ : for some $M \geq 2$ the following holds:

$$
\begin{aligned}
& \operatorname{dim}_{\mathbb{C}} \mathfrak{m}^{i} / \mathfrak{m}^{i+1}=\operatorname{dim}_{\mathbb{C}} \mathcal{P}_{i}^{N} \text { for } i=1, \ldots, M-1, \\
& \operatorname{dim}_{\mathbb{C}} \mathfrak{m}^{M} / \mathfrak{m}^{M+1}=\operatorname{dim}_{\mathbb{C}} \mathcal{P}_{M}^{N}-N
\end{aligned}
$$


where $N:=\operatorname{dim}_{\mathbb{C}} \mathfrak{m} / \mathfrak{m}^{2}$ is the embedding dimension of $\mathcal{A}$ and for every $i \geq 1$ we denote by $\mathcal{P}_{i}^{N}$ the vector space of all $i$-forms on $\mathbb{C}^{N}$ (observe that $N>0$ ). Note that the numbers $\operatorname{dim}_{\mathbb{C}} \mathcal{P}_{i}^{N}$ are well-known:

$$
\operatorname{dim}_{\mathbb{C}} \mathcal{P}_{i}^{N}=\left(\begin{array}{c}
i+N-1 \\
i
\end{array}\right) .
$$

REMARK 2.1. Verification of conditions $(*)_{2}$ and $(*)_{3}$ for a given algebra $\mathcal{A}$ is not hard. Indeed, $(*)_{2}$ means that

$$
\operatorname{dim}_{\mathbb{C}} H_{1}\left(K_{f_{1}, \ldots, f_{N}}\right)=N
$$

where $K_{f_{1}, \ldots, f_{N}}$ is the Koszul complex constructed from a basis $f_{1}, \ldots, f_{N}$ in a complement to $\mathfrak{m}^{2}$ in $\mathfrak{m}$ (see, e.g. pp. 169-172 in [Ma]); computing the left-hand side of (2.1) is straightforward from the definitions. Further, computation of all the dimensions $\operatorname{dim}_{\mathbb{C}} \mathfrak{m}^{i} / \mathfrak{m}^{i+1}$ required for verifying $(*)_{3}$ is easily done as well. In contrast, verification of condition $(*)_{1}$ may be quite difficult, especially if one is interested in constructing a standard grading explicitly. For example, to deal with condition $(*)_{1}$ one can first check whether $\operatorname{Gr}(\mathcal{A})$ is Gorenstein and, if this is indeed the case, use the criterion for isomorphism of Gorenstein algebras obtained in [FIKK] to see whether $\mathcal{A}$ and $\operatorname{Gr}(\mathcal{A})$ are isomorphic. We note that a necessary condition for the existence of a standard grading on a Gorenstein algebra is given in Proposition 8.1 in [FK].

We are now ready to state our result.

THEOREM 2.2. Let $\mathcal{A}$ be a local unital complex commutative associative algebra with $1<\operatorname{dim}_{\mathbb{C}} \mathcal{A}<\infty$. Then $\mathcal{A}$ is isomorphic to the algebra $\mathcal{A}_{\mathbf{P}}$ for some $\mathbf{P}$ if and only if $\mathcal{A}$ has Property $(*)$, in which case $N=n$ and $M=m=\nu / n+1$.

Proof. First, we let $\mathcal{A}=\mathcal{A}_{\mathbf{P}}$ for some map $\mathbf{P}$ as above and show that $\mathcal{A}$ has Property $(*)$. Indeed, $p_{1}, \ldots, p_{n}$ is a regular sequence in $\mathcal{O}_{n}$ (see Theorem 2.1.2 in $[\mathrm{BH}]$ ), hence $\mathcal{A}$ satisfies $(*)_{2}$. Next, we clearly have $N=n$, and for any $i \geq 0$ define $\mathcal{L}_{i}$ to be the linear subspace of $\mathcal{A}$ that consists of all elements represented by germs of forms in $\mathcal{P}_{i}^{n}$. These subspaces form a standard grading on $\mathcal{A}$, hence $(*)_{1}$ is satisfied. Further, condition $(*)_{3}$ obviously holds with $M=m$. Finally, as we noted in [IK], the embedding dimension $n$ divides $\nu$ and one has $m=\nu / n+1$.

Conversely, let $\mathcal{A}$ be a local unital complex commutative associative algebra with $1<\operatorname{dim}_{\mathbb{C}} \mathcal{A}<\infty$ having Property $(*)$. Choose a basis $f_{1}, \ldots, f_{N}$ in a complement to $\mathfrak{m}^{2}$ in $\mathfrak{m}$. The elements $f_{1}, \ldots, f_{N}$ generate $\mathcal{A}$ (as an algebra), hence $\mathcal{A}$ is isomorphic to $\mathbb{C}\left[z_{1}, \ldots, z_{N}\right] / R$, where $R$ is the ideal of all relations among $f_{1}, \ldots, f_{N}$, i.e. polynomials $P \in \mathbb{C}\left[z_{1}, \ldots, z_{N}\right]$ with $P\left(f_{1}, \ldots, f_{N}\right)=0$. Observe that $R$ contains all homogeneous polynomials of degree greater than $\nu$. It then follows that $\mathcal{A}$ is isomorphic to $\mathcal{O}_{N} / R_{0}$, where $R_{0}$ is the ideal in $\mathcal{O}_{N}$ generated by the germs of all relations.

We will now use condition $(*)_{1}$. Namely, choosing $f_{1}, \ldots, f_{N}$ to be a basis in $\mathcal{L}_{1}$, we see that $R$ is generated by finitely many homogeneous relations, which yields $R_{0} \subset \mathfrak{m}_{N}^{2}$, where $\mathfrak{m}_{N}$ is the maximal ideal of $\mathcal{O}_{N}$. Next, since $\mathcal{A}$ is isomorphic to the quotient of the regular local ring $\mathcal{O}_{N}$ by the ideal $R_{0} \subset \mathfrak{m}_{N}^{2}$, condition $(*)_{2}$ means that the minimal number of generators of $R_{0}$ is equal to $N$. Further, condition $(*)_{3}$ means that the ideal $R$ contains no relations of degree less than $M$ and that the linear subspace $\mathcal{H} \subset R$ of all homogeneous relations of degree $M$ has dimension $N$. 
Choose a set of generators of $R_{0}$ consisting of $N$ elements, let $g_{1}, \ldots, g_{N}$ be holomorphic functions representing the generators, and fix a basis $p_{1}, \ldots, p_{N}$ of $\mathcal{H}$. Then in a neighborhood of the origin we have

$$
p_{i}=\sum_{j=1}^{N} a_{i j} g_{j}, \quad i=1, \ldots, N
$$

for some holomorphic functions $a_{i j}$. On the other hand, if we complete the basis $p_{1}, \ldots, p_{N}$ to a set of generators of $R$ by adding homogeneous elements $q_{1}, \ldots, q_{L} \in R$ of degree greater than $M$, each function $g_{j}$ can be written as

$$
g_{j}=\sum_{k=1}^{N} b_{j k} p_{k}+\sum_{\ell=1}^{L} c_{j \ell} q_{\ell}, \quad j=1, \ldots, N
$$

where $b_{j k}, c_{j \ell}$ are holomorphic functions. Plugging (2.3) into (2.2) and collecting terms of degree $M$ in both sides of the resulting identity, we see that the matrix $\left(a_{i j}(0)\right)$ is non-degenerate. Letting $\left(d_{j k}\right)$ be the inverse of $\left(a_{i j}\right)$ near the origin (with $\left.d_{j k}(0)=b_{j k}(0)\right)$, from equation $(2.2)$ we then obtain

$$
g_{j}=\sum_{k=1}^{N} d_{j k} p_{k}, \quad j=1, \ldots, N
$$

which implies that the germs of $p_{1}, \ldots, p_{N}$ generate $R_{0}$.

Thus, we have shown that the germs of the elements of every basis of $\mathcal{H}$ generate $R_{0}$. Let $p_{1}, \ldots, p_{N}$ be any basis and $\mathbf{P}:=\left(p_{1}, \ldots, p_{N}\right)$. By the condition $\operatorname{dim}_{\mathbb{C}} \mathcal{A}<\infty$, the map $\mathbf{P}$ is finite at the origin. We have thus proved that $\mathcal{A}$ is isomorphic to $\mathcal{A}_{\mathbf{P}}$. $\mathbf{\square}$

The proof of Theorem 2.2 provides a method for producing a map $\mathbf{P}$ from any algebra $\mathcal{A}$ having Property $(*)$, but this method requires that a standard grading on $\mathcal{A}$ be explicitly defined. As we noted in Remark 2.1, finding such a grading may be hard. On the other hand, in article [IK] we proposed an algorithm for recovering $\mathbf{P}$ up to linear equivalence from the algebra $\mathcal{A}_{\mathbf{P}}$, where one does not need to know a standard grading explicitly. Assuming that $\mathcal{A}_{\mathbf{P}}$ is given as an abstract algebra (i.e. by a multiplication table with respect to some basis), we summarize the main steps of the algorithm as follows (see [IK] for details):

1. Find $\mathfrak{m}$ and its nil-index $\nu$.

2. Determine $n$ from the formula $n=\operatorname{dim}_{\mathbb{C}} \mathfrak{m} / \mathfrak{m}^{2}$.

3. Determine $m$ from the formula $m=\nu / n+1$.

4. Choose a complement to $\mathfrak{m}^{2}$ in $\mathfrak{m}$ and an arbitrary basis $f_{1}, \ldots, f_{n}$ in this complement. 
5. Calculate $q_{1}(f), \ldots, q_{K}(f)$, where $f:=\left(f_{1}, \ldots, f_{n}\right), q_{1}(z), \ldots, q_{K}(z)$ are all monomials of degree $m$ in $z:=\left(z_{1}, \ldots, z_{n}\right)$, and $K:=\operatorname{dim}_{\mathbb{C}} \mathcal{P}_{m}^{n}$.

6. Choose a complement $\mathcal{S}$ to $\mathfrak{m}^{m+1}$ in $\mathfrak{m}^{m}$.

7. Compute $\pi\left(q_{1}(f)\right), \ldots, \pi\left(q_{K}(f)\right)$, where $\pi: \mathfrak{m}^{m} \rightarrow \mathcal{S}$ is the projection onto $\mathcal{S}$ with kernel $\mathfrak{m}^{m+1}$.

8. Find $n$ linearly independent linear relations among the vectors $\pi\left(q_{1}(f)\right), \ldots, \pi\left(q_{K}(f)\right)$ :

$\sum_{\rho=1}^{K} \gamma_{\sigma \rho} \pi\left(q_{\rho}(f)\right)=0, \quad \sigma=1, \ldots, n, \quad \gamma_{\sigma \rho} \in \mathbb{C}$.

9. The following formula then gives a map linearly equivalent to $\mathbf{P}$ : $\Phi: \mathbb{C}^{n} \rightarrow \mathbb{C}^{n}, \quad z \mapsto \Gamma q(z)$, where $\Gamma:=\left(\gamma_{\sigma \rho}\right)_{\sigma=1, \ldots, n, \rho=1, \ldots, K}$, and $q:=\left(q_{1}, \ldots, q_{K}\right)$.

REMARK 2.3. The algorithm of [IK] shows that if two algebras $\mathcal{A}_{\mathbf{P}}$ and $\mathcal{A}_{\mathbf{P}^{\prime}}$ are isomorphic, the maps $\mathbf{P}$ and $\mathbf{P}^{\prime}$ are linearly equivalent. This fact can also be seen directly by lifting an isomorphism between $\mathcal{A}_{\mathbf{P}}$ and $\mathcal{A}_{\mathbf{P}^{\prime}}$ to an automorphism of $\mathcal{O}_{n}$. Hence, the sufficiency implication of Theorem 2.2 can be strengthened as follows: for every algebra $\mathcal{A}$ having Property $(*)$ there exists exactly one, up to linear equivalence, map $\mathbf{P}$ such that $\mathcal{A}$ is isomorphic to $\mathcal{A}_{\mathbf{P}}$.

We will now apply the above algorithm to $\mathbf{P}=\mathbf{Q}:=\operatorname{grad} Q$ for a holomorphic $(m+1)$-form $Q$ on $\mathbb{C}^{n}$ with $\Delta(Q) \neq 0$. Let $\Phi$ be a map linearly equivalent to $\mathbf{Q}$ derived from the algebra $\mathcal{A}\left(\mathcal{V}_{Q}\right)$, where $\mathcal{V}_{Q}$ is the germ of the hypersurface $\{Q=0\}$ at the origin. For any $n \times n$-matrix $D$ we now introduce the holomorphic differential 1 -form $\omega^{\Phi^{D}}:=\sum_{i=1}^{n} \Phi_{i}^{D} d z_{i}$ on $\mathbb{C}^{n}$, where $\Phi_{1}^{D}, \ldots, \Phi_{n}^{D}$ are the components of the map $\Phi^{D}:=D \Phi$. Consider the equation

$$
d \omega^{\Phi^{D}}=0
$$

as a linear system with respect to the entries of the matrix $D$. It is explained in [IK] that to recover $Q$ from $\mathcal{A}\left(\mathcal{V}_{Q}\right)$ up to linear equivalence one needs to complement the above algorithm with the following two steps:

10. Find a matrix $D \in \mathrm{GL}(n, \mathbb{C})$ satisfying system $(2.4)$.

11. Integrate $\Phi^{D}$ to obtain an $(m+1)$-form linearly equivalent to $Q$.

We will now briefly sketch an example of reconstructing $Q$ from $\mathcal{A}\left(\mathcal{V}_{Q}\right)$ for the family of simple elliptic singularities of type $\widetilde{E}_{6}$. For full details on this example we refer the reader to $[\mathrm{IK}]$.

EXAmPLE 2.4. Simple elliptic $\widetilde{E}_{6}$-singularities form a family parametrized by $t \in \mathbb{C}$ satisfying $t^{3}+27 \neq 0$. Namely, for every such $t$ let $\mathcal{V}_{t}:=\mathcal{V}_{Q_{t}}$, where $Q_{t}$ is the following cubic on $\mathbb{C}^{3}$ :

$$
Q_{t}(z):=z_{1}^{3}+z_{2}^{3}+z_{3}^{3}+t z_{1} z_{2} z_{3}, \quad z:=\left(z_{1}, z_{2}, z_{3}\right)
$$

The algebra $\mathcal{A}_{t}:=\mathcal{A}\left(\mathcal{V}_{t}\right)$ has dimension 8 and with respect to a certain basis $e_{1}, \ldots, e_{8}$ 
is given by:

$$
\begin{aligned}
& e_{1} e_{j}=e_{j} \text { for } j=1, \ldots, 8, e_{2}^{2}=-\frac{t}{3} e_{3}+\frac{2 t}{3} e_{6}, e_{2} e_{3}=e_{6} \\
& e_{2} e_{4}=e_{5}-e_{6}-e_{8}, e_{2} e_{5}=e_{7}, e_{2} e_{6}=0, e_{2} e_{7}=0, e_{2} e_{8}=e_{7}, \\
& e_{3} e_{j}=0 \text { for } j=3, \ldots, 8, e_{4}^{2}=-\frac{t}{3} e_{7}, e_{4} e_{5}=e_{3}-2 e_{6}, e_{4} e_{6}=0, \\
& e_{4} e_{7}=e_{6}, e_{4} e_{8}=e_{3}-2 e_{6}, e_{5}^{2}=-\frac{t}{3} e_{5}+(2+t) e_{6}+\frac{t}{3} e_{8}, e_{5} e_{6}=0 \\
& e_{5} e_{7}=0, e_{5} e_{8}=-\frac{t}{3} e_{5}+(1+t) e_{6}+\frac{t}{3} e_{8}, e_{6} e_{j}=0 \text { for } j=6,7,8, \\
& e_{7} e_{j}=0 \text { for } j=7,8, e_{8}^{2}=-\frac{t}{3} e_{5}+t e_{6}+\frac{t}{3} e_{8} .
\end{aligned}
$$

Then the maximal ideal $\mathfrak{m}_{t}$ of $\mathcal{A}_{t}$ is spanned by $e_{2}, \ldots, e_{8}$, and we have $n=3, m=2$, $K=6$. Next, choosing $f_{1}:=e_{2}, f_{2}:=e_{4}, f_{3}:=e_{5}$ and $\mathcal{S}$ to be the linear span of $e_{3}, e_{7}, e_{5}-e_{8}$, one obtains the following three linearly independent linear relations:

$$
\pi\left(q_{1}(f)\right)+\frac{t}{3} \pi\left(q_{6}(f)\right)=0, \pi\left(q_{2}(f)\right)+\frac{t}{3} \pi\left(q_{5}(f)\right)=0, \pi\left(q_{3}(f)\right)+\frac{t}{3} \pi\left(q_{4}(f)\right)=0 .
$$

Hence

$$
\Gamma=\left(\begin{array}{cccccc}
1 & 0 & 0 & 0 & 0 & t / 3 \\
0 & 1 & 0 & 0 & t / 3 & 0 \\
0 & 0 & 1 & t / 3 & 0 & 0
\end{array}\right)
$$

which yields

$$
\Phi(z)=\left(\begin{array}{c}
z_{1}^{2}+\frac{t}{3} z_{2} z_{3} \\
z_{2}^{2}+\frac{t}{3} z_{1} z_{3} \\
z_{3}^{2}+\frac{t}{3} z_{1} z_{2}
\end{array}\right)
$$

Further, for

$$
D=\left(\begin{array}{lll}
d_{11} & d_{12} & d_{13} \\
d_{21} & d_{22} & d_{23} \\
d_{31} & d_{32} & d_{33}
\end{array}\right)
$$

system (2.4) is equivalent to the following system of equations:

$$
\begin{aligned}
& 2 d_{12}-\frac{t}{3} d_{23}=0, \quad 2 d_{21}-\frac{t}{3} d_{13}=0, \quad t d_{11}-t d_{22}=0 \\
& 2 d_{13}-\frac{t}{3} d_{32}=0, \quad 2 d_{31}-\frac{t}{3} d_{12}=0, \quad t d_{11}-t d_{33}=0 \\
& 2 d_{23}-\frac{t}{3} d_{31}=0, \quad 2 d_{32}-\frac{t}{3} d_{21}=0, \quad t d_{22}-t d_{33}=0 .
\end{aligned}
$$


If $t \neq 0$ and $t^{3} \neq 216$, the only non-degenerate solutions of (2.6) are non-zero scalar matrices. Integrating $\Phi^{D}$ for such a matrix $D$ we obtain a form proportional to $Q_{t}$. If $t=0$, any non-degenerate solution of (2.6) is a diagonal matrix with non-zero $d_{11}$, $d_{22}, d_{33}$. Integrating $\Phi^{D}$ for such a matrix $D$ we obtain the form

$$
\frac{1}{3}\left(d_{11} z_{1}^{3}+d_{22} z_{2}^{3}+d_{33} z_{3}^{3}\right)
$$

which is clearly linearly equivalent to $Q_{0}$. Finally, let $t^{3}=216$. Writing $t=6 \lambda$ with $\lambda^{3}=1$, we see that $D$ is a solution of (2.6) if and only if

$$
d_{11}=d_{22}=d_{33}, d_{12}=\lambda^{2} d_{31}, d_{23}=\lambda d_{31}, d_{21}=\lambda^{2} d_{32}, d_{13}=\lambda d_{32} .
$$

Such a matrix $D$ is non-degenerate if and only if $d_{11}^{3}+d_{31}^{3}+d_{32}^{3}-3 \lambda d_{11} d_{31} d_{32} \neq 0$. For example, letting $d_{11}=0, d_{31}=0, d_{32}=1$ one obtains

$$
\Phi^{D}=\left(\begin{array}{c}
\lambda z_{3}^{2}+2 \lambda^{2} z_{1} z_{2} \\
\lambda^{2} z_{1}^{2}+2 z_{2} z_{3} \\
z_{2}^{2}+2 \lambda z_{1} z_{3}
\end{array}\right) .
$$

Integration of $\Phi^{D}$ leads to the form

$$
\mathcal{Q}_{\lambda}:=\lambda^{2} z_{1}^{2} z_{2}+\lambda z_{1} z_{3}^{2}+z_{2}^{2} z_{3},
$$

and using invariant theory one can verify that $\mathcal{Q}_{\lambda}$ is indeed linearly equivalent to $Q_{t}$.

3. The second method. Let $\mathcal{A}$ be a complex Gorenstein algebra with $2<$ $\operatorname{dim}_{\mathbb{C}} \mathcal{A}<\infty$ and maximal ideal $\mathfrak{m}$. Define $\exp _{2}: \mathfrak{m} \rightarrow \mathfrak{m}$ to be the map

$$
\exp _{2}(x):=\sum_{s=2}^{\nu} \frac{1}{s !} x^{s}, \quad x \in \mathfrak{m},
$$

where, as before, $\nu \geq 2$ is the nil-index of $\mathfrak{m}$. Using the map $\exp _{2}$, one can associate to the algebra $\mathcal{A}$ a collection of polynomials of a special form. Let $\Pi$ be a hyperplane in $\mathfrak{m}$ complementary to $\operatorname{Ann}(\mathfrak{m})$. A $\mathbb{C}$-valued polynomial $P$ on $\Pi$ is called a nil-polynomial if there exists a linear form $\omega: \mathfrak{m} \rightarrow \mathbb{C}$ such that $\operatorname{ker} \omega=\Pi$ and $P=\left.\omega \circ \exp _{2}\right|_{\Pi}$. Observe that $\operatorname{deg} P=\nu$.

If $P_{1}, P_{2}$ are two nil-polynomials arising from Gorenstein algebras $\mathcal{A}_{1}, \mathcal{A}_{2}$ and hyperplanes $\Pi_{1} \subset \mathfrak{m}_{1}, \Pi_{2} \subset \mathfrak{m}_{2}$, respectively, then $P_{1}, P_{2}$ are called linearly equivalent if there exist $c \in \mathbb{C}^{*}$ and a linear isomorphism $L: \Pi_{1} \rightarrow \Pi_{2}$ such that $c P_{1}=P_{2} \circ L$. It then follows from results of [FIKK] (see also [FK], [I1], [I3]) that the map $\varphi$ : $\mathfrak{m}_{1} \rightarrow \mathfrak{m}_{2}, \varphi(x+y):=L(x)+c \widetilde{\omega}_{2}^{-1}\left(\omega_{1}(y)\right)$, is an algebra isomorphism, where $x \in \Pi_{1}$, $y \in \operatorname{Ann}\left(\mathfrak{m}_{1}\right), \widetilde{\omega}_{2}:=\left.\omega_{2}\right|_{\operatorname{Ann}\left(\mathfrak{m}_{2}\right)}$, and $\omega_{1}, \omega_{2}$ are the linear forms corresponding to $P_{1}, P_{2}$, respectively. Thus, if $P_{1}, P_{2}$ are linearly equivalent, the algebras $\mathcal{A}_{1}, \mathcal{A}_{2}$ are isomorphic.

A nil-polynomial $P=\left.\omega \circ \exp _{2}\right|_{\Pi}$ arising from a Gorenstein algebra $\mathcal{A}$ extends to the polynomial $\widetilde{P}:=\omega \circ \exp _{2}$ on all of $\mathfrak{m}$. Let $\widetilde{P}^{[s]}$ be the homogeneous component of order $s$ of $\widetilde{P}$, with $s=2, \ldots, \nu$. One has $\widetilde{P}^{[s]}(y)=0, \widetilde{P}^{[s]}(x+y)=\widetilde{P}^{[s]}(x)$ for all $x \in \mathfrak{m}, y \in \mathfrak{m}^{\nu+2-s}$. Thus, $\widetilde{P}^{[s]}$ gives rise to a polynomial $P^{[s]}$ on the quotient $\mathfrak{m} / \mathfrak{m}^{\nu+2-s}$. It then follows that if two nil-polynomials $P_{1}, P_{2}$ arising from Gorenstein 
algebras $\mathcal{A}_{1}, \mathcal{A}_{2}$, respectively, are linearly equivalent, there exist $c \in \mathbb{C}^{*}$ and algebra isomorphisms $L^{[s]}: \mathfrak{m}_{1} / \mathfrak{m}_{1}^{\nu+2-s} \rightarrow \mathfrak{m}_{2} / \mathfrak{m}_{2}^{\nu+2-s}$ such that $c P_{1}^{[s]}=P_{2}^{[s]} \circ L^{[s]}$, where $\nu$ is the nil-index of each of $\mathfrak{m}_{1}, \mathfrak{m}_{2}$ (note that the nil-indices of $\mathfrak{m}_{1}, \mathfrak{m}_{2}$ coincide since $\operatorname{deg} P_{1}=\operatorname{deg} P_{2}$ ). Observe also that for any nil-polynomials $P$ and $P^{\prime}$ arising from the same algebra the corresponding highest-order components $P^{[\nu]}$ and $P^{\prime}[\nu]$ coincide up to scale.

Next, recall that the algebra $\mathcal{A}$ is (non-negatively) graded if it can be represented as $\mathcal{A}=\oplus_{i \geq 0} \mathcal{L}_{i}$, where $\mathcal{L}_{i}$ are linear subspaces of $\mathcal{A}$ with $\mathcal{L}_{0} \simeq \mathbb{C}$ and $\mathcal{L}_{i} \mathcal{L}_{j} \subset \mathcal{L}_{i+j}$ for all $i, j$. In this case $\mathfrak{m}=\oplus_{i>0} \mathcal{L}_{i}$ and $\operatorname{Ann}(\mathfrak{m})=\mathcal{L}_{d}$ for $d:=\max \left\{i: \mathcal{L}_{i} \neq 0\right\}$. It is shown in [FIKK] (see also [FK], [I1]) that if $\mathcal{A}$ is graded, then all nil-polynomials arising from $\mathcal{A}$ are linearly equivalent. Thus, the following theorem holds.

THEOREM 3.1. [EI] Let $\mathcal{A}_{1}, \mathcal{A}_{2}$ be graded Gorenstein algebras with $2<\operatorname{dim}_{\mathbb{C}} \mathcal{A}_{j}<\infty, j=1,2$, and $P_{1}, P_{2}$ some nil-polynomials arising from $\mathcal{A}_{1}, \mathcal{A}_{2}$, respectively. Assume that $\mathcal{A}_{1}, \mathcal{A}_{2}$ are isomorphic. Then there exist $c \in \mathbb{C}^{*}$ and algebra isomorphisms $L^{[s]}: \mathfrak{m}_{1} / \mathfrak{m}_{1}^{\nu+2-s} \rightarrow \mathfrak{m}_{2} / \mathfrak{m}_{2}^{\nu+2-s}$ such that $c P_{1}^{[s]}=P_{2}^{[s]} \circ L^{[s]}$ for $s=2, \ldots, \nu$.

Theorem 3.1 allows one to utilize classical invariant theory for constructing certain numerical invariants of graded Gorenstein algebras. We will now recall the definitions of relative and absolute classical invariants (see, e.g. [O] for details). These definitions can be given in a coordinate-free setting.

Let $W$ be a finite-dimensional complex vector space and $\mathcal{Q}_{W}^{m+1}$ the linear space of holomorphic forms of degree $m+1$ on $W$, with $m \geq 1$. Define an action of GL(W) on $\mathcal{Q}_{W}^{m+1}$ by the formula

$$
(C, Q) \mapsto Q_{C}, Q_{C}(w):=Q\left(C^{-1} w\right), \text { where } C \in \mathrm{GL}(W), Q \in \mathcal{Q}_{W}^{m+1}, w \in W .
$$

An invariant (or relative classical invariant) of forms of degree $m+1$ on $W$ is a polynomial $I: \mathcal{Q}_{W}^{m+1} \rightarrow \mathbb{C}$ such that for any $Q \in \mathcal{Q}_{W}^{m+1}$ and any $C \in \mathrm{GL}(W)$ one has $I(Q)=(\operatorname{det} C)^{k} I\left(Q_{C}\right)$, where $k$ is a non-negative integer called the weight of $I$. It follows that $I$ is in fact homogeneous of degree $k \operatorname{dim}_{\mathbb{C}} W /(m+1)$. Finite sums of relative invariants comprise the algebra of polynomial $\mathrm{SL}(W)$-invariants of $\mathcal{Q}_{W}^{m+1}$, called the algebra of invariants (or algebra of classical invariants) of forms of degree $m+1$ on $W$. By the Hilbert Basis Theorem, this algebra is finitely generated. For any two invariants $I$ and $\widetilde{I}$, with $\widetilde{I} \not \equiv 0$, the ratio $I / \widetilde{I}$ yields a rational function on $\mathcal{Q}_{W}^{m+1}$ that is defined, in particular, at the points where $\widetilde{I}$ does not vanish. If $I$ and $\widetilde{I}$ have equal weights, this function does not change under the action of $\operatorname{GL}(W)$, and we say that $I / \widetilde{I}$ is an absolute invariant (or absolute classical invariant) of forms of degree $m+1$ on $W$.

If one fixes coordinates $z_{1}, \ldots, z_{n}$ in $W$, then $W$ is identified with $\mathbb{C}^{n}, \mathrm{GL}(W)$ with $\operatorname{GL}(n, \mathbb{C})$, and any element $Q \in \mathcal{Q}_{W}^{m+1}$ is written as a homogeneous polynomial of degree $m+1$ in $z_{1}, \ldots, z_{n}$, which is the representation of $(m+1)$-forms that we have utilized in the paper so far. Invariants are usually defined in terms of the coefficients of the polynomial in $z_{1}, \ldots, z_{n}$ representing $Q$ (see, e.g. the definition of $\Delta$ in [GKZ]). Observe, however, that for any absolute invariant $\mathcal{I}$ its value $\mathcal{I}(Q)$ is in fact independent of the choice of coordinates in $W$.

Now, Theorem 3.1 yields the following result.

THEOREM 3.2. [EI] Let $\mathcal{A}$ be a graded Gorenstein algebra such that $2<\operatorname{dim}_{\mathbb{C}} \mathcal{A}<\infty$, and $P$ a nil-polynomial arising from $\mathcal{A}$. Further, for a fixed 
$s \in\{2, \ldots, \nu\}$, let $W$ be a complex vector space isomorphic to $\mathfrak{m} / \mathfrak{m}^{\nu+2-s}$ by means of a linear map $\psi: W \rightarrow \mathfrak{m} / \mathfrak{m}^{\nu+2-s}$. Fix an absolute invariant $\mathcal{I}$ of forms of degree $s$ on $W$. Then the value $\mathcal{I}\left(\psi^{*} P^{[s]}\right)$ depends only on the isomorphism class of $\mathcal{A}$.

Let $\mathcal{V}$ be a hypersurface germ having a homogeneous singularity. Consider the moduli algebra $\mathcal{A}(\mathcal{V})$ of $\mathcal{V}$, and let $\mathfrak{m}(\mathcal{V})$ be the maximal ideal of $\mathcal{A}(\mathcal{V})$. Theorem 3.2 allows one to explicitly extract from $\mathcal{A}(\mathcal{V})$ invariants of $\mathcal{V}$ of the form $\mathcal{I}\left(P^{[s]}\right)$, where $P$ is a nil-polynomial arising from $\mathcal{A}(\mathcal{V})$ and $\mathcal{I}$ is an absolute invariant of forms of degree $s$ on $\mathfrak{m}(\mathcal{V}) / \mathfrak{m}(\mathcal{V})^{\nu+2-s}$, with $s=2, \ldots, \nu$. It is natural to ask whether these invariants form a complete set, i.e. whether they solve the biholomorphic equivalence problem for hypersurface germs with homogeneous singularity. We attempted to answer this question in [EI], as described below.

For $m \geq 2$ define

$$
X_{n}^{m+1}:=\left\{Q \in \mathcal{Q}_{\mathbb{C}^{n}}^{m+1}: \Delta(Q) \neq 0\right\} .
$$

Any hypersurface germ $\mathcal{V}$ with homogeneous singularity at the origin in $\mathbb{C}^{n}$ and $\operatorname{dim}_{\mathbb{C}} \mathcal{A}(\mathcal{V})>1$ is biholomorphic to some $\mathcal{V}_{Q}$ with $Q \in X_{n}^{m+1}, m \geq 2$. First of all, we need the following fact.

Proposition 3.3. [EI $]^{1}$ The orbits of the $\mathrm{GL}(n, \mathbb{C})$-action on $X_{n}^{m+1}$ are separated by absolute invariants of the kind

$$
\mathcal{I}=\frac{I}{\Delta^{p}}
$$

where $p$ is a non-negative integer and $I$ is a relative classical invariant.

In what follows we denote by $\mathcal{I}_{n}^{m+1}$ the algebra of restrictions to $X_{n}^{m+1}$ of absolute invariants of the form (3.1). By the Hilbert Basis Theorem, this algebra is finitely generated. Observe that $\mathcal{I}_{n}^{m+1}$ is exactly the algebra of $\operatorname{GL}(n, \mathbb{C})$-invariant regular functions on the affine algebraic variety $X_{n}^{m+1}$.

As we noted in [IK], if $Q$ is a form in $X_{n}^{m+1}$ with $m \geq 2$, then for the nil-index $\nu$ of the maximal ideal $\mathfrak{m}\left(\mathcal{V}_{Q}\right)$ of the moduli algebra $\mathcal{A}\left(\mathcal{V}_{Q}\right)$ one has $\nu=n(m-1)$. Furthermore, $\operatorname{dim}_{\mathbb{C}} \mathfrak{m}\left(\mathcal{V}_{Q}\right) / \mathfrak{m}\left(\mathcal{V}_{Q}\right)^{2}=n$. Thus, every nil-polynomial $P$ arising from $\mathcal{A}\left(\mathcal{V}_{Q}\right)$ has degree $n(m-1)$, and the corresponding polynomial $P^{[n(m-1)]}$ is a form on an $n$-dimensional space. For any two nil-polynomials $P, P^{\prime}$ the forms $P^{[n(m-1)]}$, $P^{\prime}[n(m-1)]$ coincide up to scale, and we say that any of the mutually proportional forms of degree $n(m-1)$ arising in this way is associated to $Q$. Thus, upon identification of $\mathfrak{m}\left(\mathcal{V}_{Q}\right) / \mathfrak{m}\left(\mathcal{V}_{Q}\right)^{2}$ with $\mathbb{C}^{n}$, every invariant of $\mathcal{V}_{Q}$ provided by Theorem 3.2 for $s=\nu$ is given as $\widetilde{\mathcal{I}}(\widetilde{Q})$, where $\widetilde{\mathcal{I}}$ is an absolute invariant of forms of degree $n(m-1)$ on $\mathbb{C}^{n}$ and $\widetilde{Q}$ is a form associated to $Q$. Note that invariants of this kind were considered in article [Ea] (cf. p. 401 in [Em]), where associated forms were introduced in slightly different terms. In [EI] we proposed a conjecture similar to (but a priori weaker than) the following one.

CONJECTURE 3.4. For any $\mathcal{I} \in \mathcal{I}_{n}^{m+1}$ there exists an absolute invariant $\widetilde{\mathcal{I}}$ of forms of degree $n(m-1)$ on $\mathbb{C}^{n}$ such that for all $Q \in X_{n}^{m+1}$ the following holds: upon identification of $\mathfrak{m}\left(\mathcal{V}_{Q}\right) / \mathfrak{m}\left(\mathcal{V}_{Q}\right)^{2}$ with $\mathbb{C}^{n}$ the invariant $\widetilde{\mathcal{I}}$ is defined at some (hence at every) form $\widetilde{Q}$ associated to $Q$ and $\widetilde{\mathcal{I}}(\widetilde{Q})=\mathcal{I}(Q)$.

\footnotetext{
${ }^{1}$ The proof of this proposition given in $[\mathrm{EI}]$ has been suggested to us by A. Gorinov.
} 
In order to discuss one implication of this conjecture, let us canonically identify $\mathfrak{m}\left(\mathcal{V}_{Q}\right) / \mathfrak{m}\left(\mathcal{V}_{Q}\right)^{2}$ with $\mathbb{C}^{n}$. Namely, let $Z_{j}$ be the element of $\mathfrak{m}\left(\mathcal{V}_{Q}\right)$ represented by the germ of the coordinate function $z_{j}$, and $e_{j}$ the element of $\mathfrak{m}\left(\mathcal{V}_{Q}\right) / \mathfrak{m}\left(\mathcal{V}_{Q}\right)^{2}$ represented by $Z_{j}, j=1, \ldots, n$. Denote by $w_{1}, \ldots, w_{n}$ the coordinates in $\mathfrak{m}\left(\mathcal{V}_{Q}\right) / \mathfrak{m}\left(\mathcal{V}_{Q}\right)^{2}$ with respect to the basis $e_{1}, \ldots, e_{n}$. Now, for an absolute invariant $\widetilde{\mathcal{I}}$ of forms of degree $n(m-1)$ in the variables $w_{1}, \ldots, w_{n}$ it is easy to observe that $\widetilde{\mathcal{I}}(\widetilde{Q})$ is rational when regarded as a function of $Q$, with $\widetilde{Q}$ associated to $Q \in X_{n}^{m+1}$, provided this function is defined at least at one point of $X_{n}^{m+1}$. Let $\mathcal{R}_{n}^{m+1}$ denote the collection of all invariant rational functions on $X_{n}^{m+1}$ obtained in this way. Further, let $\widehat{\mathcal{I}}_{n}^{m+1}$ be the algebra of restrictions to $X_{n}^{m+1}$ of all absolute invariants of forms of degree $m+1$ on $\mathbb{C}^{n}$. Note that $\mathcal{R}_{n}^{m+1}$ lies in $\widehat{\mathcal{I}}_{n}^{m+1}$ (see Proposition 1 in [DC]). We now observe that Conjecture 3.4 implies

$$
\mathcal{R}_{n}^{m+1}=\widehat{\mathcal{I}}_{n}^{m+1} .
$$

Indeed, since every element of $\widehat{\mathcal{I}}_{n}^{m+1}$ can be represented as a ratio of two elements of $\mathcal{I}_{n}^{m+1}$ (see Proposition 6.2 in [Mu]), identity (3.2) is equivalent to the inclusion $\mathcal{I}_{n}^{m+1} \subset \mathcal{R}_{n}^{m+1}$, which clearly follows from Conjecture 3.4 (cf. [EI], [I2]).

We remark that identity (3.2) is interesting from the invariant-theoretic point of view, since it means that the invariant theory of forms of degree $m+1$ naturally embeds into that of forms of degree $n(m-1)$. Observe that $(3.2)$ is a priori weaker than Conjecture 3.4 since it may happen that for some $\mathcal{I} \in \mathcal{I}_{n}^{m+1}$ there exist an absolute invariant $\widetilde{\mathcal{I}}$ of forms of degree $n(m-1)$ on $\mathbb{C}^{n}$ such that $\widetilde{\mathcal{I}}(\widetilde{Q}) \equiv \mathcal{I}(Q)$, where $\widetilde{\mathcal{I}}(\widetilde{Q})$ is regarded as a function of $Q$, but for some $Q_{0} \in X_{n}^{m+1}$ the invariant $\widetilde{\mathcal{I}}$ is not defined at the forms associated to $Q_{0}$.

For binary quartics $(n=2, m=3)$ and ternary cubics $(n=3, m=2)$ the conjecture was essentially verified in [Ea] (see also [EI]). Furthermore, in [EI] we showed that the conjecture also holds for binary quintics $(n=2, m=4)$ and binary sextics $(n=2, m=5)$. If the conjecture were confirmed in full generality, together with Proposition 3.3 it would imply that any form $Q \in X_{n}^{m+1}$ can be reconstructed, in principle, up to linear equivalence from any of its associated forms by way of computing absolute invariants. In particular, the invariants supplied by Theorem 3.2 for $s=\nu$ would be shown to form a complete system in the case of hypersurface germs with homogeneous singularity. However, to explicitly recover $Q$ from an associated form $\widetilde{Q}$ one would also need to determine $m$ (as in Step 3 in Section 2) and to do the following:

(a) Find some generators $\mathcal{I}_{1}, \ldots, \mathcal{I}_{L}$ of $\mathcal{I}_{n}^{m+1}$.

(b) Find absolute invariants $\widetilde{\mathcal{I}}_{1}, \ldots, \widetilde{\mathcal{I}}_{L}$ of forms of degree $n(m-1)$ on $\mathbb{C}^{n}$ such that $\widetilde{\mathcal{I}}_{i}(\widetilde{Q}) \equiv \mathcal{I}_{i}(Q)$ for all $i$.

(c) Solve the following "fitting problem": find a form in $X_{n}^{m+1}$ for which the invariants $\mathcal{I}_{1}, \ldots, \mathcal{I}_{L}$ take prescribed values.

Each of tasks (a)-(c) is rather hard, and therefore this method for recovering $Q$ from $\mathcal{A}\left(\mathcal{V}_{Q}\right)$ is far less explicit than the one described in Section 2, even if Conjecture 3.4 is assumed to hold. However, if one discovers a way for extracting $Q$ from $\widetilde{Q}$ that avoids computing classical invariants, the present method may become rather useful as well. Indeed, as we will now see, computing associated forms from the algebra $\mathcal{A}\left(\mathcal{V}_{Q}\right)$ is quite easy. 
Suppose that $\mathcal{A}\left(\mathcal{V}_{Q}\right)$ is given as an abstract associative algebra and, as in Step 1 in Section 2, find the maximal ideal $\mathfrak{m}\left(\mathcal{V}_{Q}\right)$ and its nil-index $\nu$. Next, choose a basis $f_{1}, \ldots, f_{n}$ in a complement to $\mathfrak{m}\left(\mathcal{V}_{Q}\right)^{2}$ in $\mathfrak{m}\left(\mathcal{V}_{Q}\right)$, and let $\mathbf{f}_{j}$ be the element of $\mathfrak{m}\left(\mathcal{V}_{Q}\right) / \mathfrak{m}\left(\mathcal{V}_{Q}\right)^{2}$ represented by $f_{j}$ for $j=1, \ldots, n$. Denote by $\zeta_{1}, \ldots, \zeta_{n}$ the coordinates in $\mathfrak{m}\left(\mathcal{V}_{Q}\right) / \mathfrak{m}\left(\mathcal{V}_{Q}\right)^{2}$ with respect to the basis $\mathbf{f}_{1}, \ldots, \mathbf{f}_{n}$. Further, choose a vector $f_{0}$ spanning $\operatorname{Ann}\left(\mathfrak{m}\left(\mathcal{V}_{Q}\right)\right)$. If $k_{1}, \ldots, k_{n}$ are non-negative integers such that $k_{1}+\cdots+k_{n}=\nu$, the product $f_{1}^{k_{1}} \ldots f_{n}^{k_{n}}$ is an element of $\operatorname{Ann}\left(\mathfrak{m}\left(\mathcal{V}_{Q}\right)\right)$, and thus we have $f_{1}^{k_{1}} \ldots f_{n}^{k_{n}}=\mu_{k_{1}, \ldots, k_{n}} f_{0}$ for some $\mu_{k_{1}, \ldots, k_{n}} \in \mathbb{C}$. Then the form

$$
\sum_{k_{1}+\cdots+k_{n}=\nu} \mu_{k_{1}, \ldots, k_{n}}\left(\begin{array}{c}
\nu \\
k_{1}, \ldots, k_{n}
\end{array}\right) \zeta_{1}^{k_{1}} \ldots \zeta_{n}^{k_{n}}
$$

is a coordinate representation of a form associated to $Q$, where

$$
\left(\begin{array}{c}
\nu \\
k_{1}, \ldots, k_{n}
\end{array}\right):=\frac{\nu !}{k_{1} ! \ldots k_{n} !}
$$

is a multinomial coefficient. Clearly, the computations required to find form (3.4) are much easier than those needed for our first method.

As in Section 2, we will now illustrate our second method for reconstructing $Q$ from $\mathcal{A}\left(\mathcal{V}_{Q}\right)$ by the example of simple elliptic singularities of type $\widetilde{E}_{6}$. Note that any form associated to a ternary cubic is again a ternary cubic and that Conjecture 3.4 has been confirmed in this case (see [Ea], [EI]).

EXAMPLE 3.5. We use the notation introduced in Example 2.4 and assume, as before, that the algebra $\mathcal{A}_{t}$ is given by multiplication table (2.5). Further, as before, choose the following basis in a complement to $\mathfrak{m}_{t}^{2}$ in $\mathfrak{m}_{t}: f_{1}:=e_{2}, f_{2}:=e_{4}, f_{3}:=e_{5}$, and also let $f_{0}:=e_{6}$. Then for the coefficients in formula (3.4) table (2.5) implies

$$
\mu_{3,0,0}=\mu_{0,3,0}=\mu_{0,0,3}=-\frac{t}{3}, \quad \mu_{1,1,1}=1,
$$

with all the remaining $\mu_{k_{1}, k_{2}, k_{3}}$ being zero. Then formula (3.4) yields the following form associated to $Q_{t}$ :

$$
\widetilde{Q}_{t}:=-\frac{t}{3}\left(\zeta_{1}^{3}+\zeta_{2}^{3}+\zeta_{3}^{3}\right)+6 \zeta_{1} \zeta_{2} \zeta_{3}
$$

(cf. $[\mathrm{Ea}],[\mathrm{Em}])$.

Further, as in Example 2.4, we see that $m=2$. Fortunately, for $n=3, m=2$ tasks (a)-(c) stated in (3.3) are well-understood. First, we discuss task (a). The algebra of classical invariants of ternary cubics is generated by certain invariants $\mathrm{I}_{4}$ and $I_{6}$, where the subscripts indicate the degrees (see pp. 381-389 in [Ell]). For a ternary cubic of the form

$$
Q(z)=a z_{1}^{3}+b z_{2}^{3}+c z_{3}^{3}+6 d z_{1} z_{2} z_{3}
$$

the values of $I_{4}$ and $I_{6}$ are computed as follows:

$$
\mathrm{I}_{4}(Q)=a b c d-d^{4}, \quad \mathrm{I}_{6}(Q)=a^{2} b^{2} c^{2}-20 a b c d^{3}-8 d^{6},
$$

and $\Delta(Q)=\mathrm{I}_{6}^{2}+64 \mathrm{I}_{4}^{3} \cdot{ }^{2}$ Define an absolute invariant of ternary cubics as

$$
\mathrm{J}:=\frac{\mathrm{I}_{4}^{3}}{\Delta}
$$

\footnotetext{
${ }^{2}$ This formula for the discriminant of a ternary cubic differs from the general formula given in [GKZ] by a scalar factor.
} 
It is easy to see that the restriction $\left.\mathrm{J}\right|_{X_{3}^{3}}$ generates the algebra $\mathcal{I}_{3}^{3}$. In particular, any two non-equivalent ternary cubics with non-vanishing discriminant are distinguished by $\mathrm{J}$ (see Proposition 3.3). Further, we have

$$
\mathrm{J}\left(Q_{t}\right)=-\frac{t^{3}\left(t^{3}-216\right)^{3}}{110592\left(t^{3}+27\right)^{3}} .
$$

Note that $\mathrm{J}\left(Q_{t}\right)=j\left(Z_{t}\right) / 110592$, where $j\left(Z_{t}\right)$ is the value of the $j$-invariant for the elliptic curve $Z_{t}$ in $\mathbb{C P}^{2}$ defined by $Q_{t}$. Details on computing $\mathrm{J}(Q)$ for any ternary cubic $Q$ with $\Delta(Q) \neq 0$ can be found, for example, in [Ea]. These calculations can be performed on a computer using, for instance, the Singular package (see [DGPS], $[\mathrm{MMN}])$.

Next, regarding task (b), consider the following absolute invariant of ternary cubics:

$$
\mathrm{K}:=\frac{1}{4096 \mathrm{~J}}
$$

A straightforward computation shows that $\mathrm{K}\left(\widetilde{Q}_{t}\right)=\mathrm{J}\left(Q_{t}\right)$.

Finally, we deal with task (c), i.e. fix $a \in \mathbb{C}$ and attempt to find a ternary cubic $Q$ with $\Delta(Q) \neq 0$ for which one has $\mathrm{J}(Q)=a$. Every ternary cubic with non-vanishing discriminant is linearly equivalent to $Q_{s}$ for some $s \in \mathbb{C}, s^{3}+27 \neq 0$ (see p. 401 in $[\mathrm{W}]$ ), hence by (3.5) task (c) is equivalent to the problem of finding $s$ that satisfies

$$
-\frac{s^{3}\left(s^{3}-216\right)^{3}}{110592\left(s^{3}+27\right)^{3}}=a .
$$

Since (3.6) is an equation of degree four with respect to $s^{3}$, it can be solved explicitly in terms of $a$. If $s_{0}$ is a solution to (3.6) with $a=\mathrm{K}\left(\widetilde{Q}_{t}\right)$, then the form $Q_{s_{0}}$ is linearly equivalent to $Q_{t}$ as required.

\section{REFERENCES}

[B] H. BAss, On the ubiquity of Gorenstein rings, Math. Z., 82 (1963), pp. 8-28.

[BH] W. Bruns and J. Herzog, Cohen-Macaulay Rings, Cambridge Studies in Advanced Mathematics 39, Cambridge University Press, Cambridge, 1993.

[DGPS] W. Decker, G.-M. Greuel, G. Pfister, and H. Schönemann, Singular 3-1-3, a computer algebra system for polynomial computations, http://www.singular.uni-kl.de, 2011.

[DC] J. A. Dieudonné And J. B. Carrell, Invariant theory, old and new, Adv. in Math., 4 (1970), pp. 1-80.

[Ea] M. G. EAstwood, Moduli of isolated hypersurface singularities, Asian J. Math., 8 (2004), pp. 305-313.

[EI] M. G. EASTwOOD AND A. V. ISAEV, Extracting invariants of isolated hypersurface singularities from their moduli algebras, Math. Ann., 356 (2013), pp. 73-98.

[Ell] E. B. Elliotr, An Introduction to the Algebra of Quantics, Oxford University Press, 1895.

[Em] J. Emsalem, Géométrie des points épais, Bull. Soc. Math. France, 106 (1978), pp. 399416.

[FIKK] G. Fels, A. Isaev, W. Kaup, And N. KRuzhilin, Isolated hypersurface singularities and special polynomial realizations of affine quadrics, J. Geom. Analysis, 21 (2011), pp. $767-782$.

[FK] G. FELS AND W. KAUP, Nilpotent algebras and affinely homogeneous surfaces, Math. Ann., 353 (2012), pp. 1315-1350. 
[GKZ] I. M. Gelfand, M. M. Kapranov, and A. V. Zelevinsky, Discriminants, Resultants and Multidimensional Determinants, Modern Birkhäuser Classics, Birkhäuser Boston, Inc., Boston, MA, 2008.

[GLS] G.-M. Greuel, C. Lossen, And E. Shustin, Introduction to Singularities and Deformations, Springer Monographs in Mathematics, Springer, Berlin, 2007.

[H] C. Huneke, Hyman Bass and ubiquity: Gorenstein rings, in "Algebra, K-theory, Groups, and Education" (New York, 1997), Contemp. Math. 243, Amer. Math. Soc., Providence, RI, 1999, pp. 55-78.

[I1] A. V. IsAEv, On the affine homogeneity of algebraic hypersurfaces arising from Gorenstein algebras, Asian J. Math., 15 (2011), pp. 631-640.

[I2] A. V. IsAev, Application of classical invariant theory to biholomorphic classification of plane curve singularities, and associated binary forms, Proc. Steklov Inst. Math., 279 (2012), pp. 245-256.

[I3] A. V. IsAEV, On a new criterion for isomorphism of Gorenstein algebras, preprint, available from http://arxiv.org/abs/1201.6100.

[IK] A. V. IsAev AND N. G. KRUZhiLin, Explicit reconstruction of homogeneous isolated hypersurface singularities from their Milnor algebras, Proc. Amer. Math. Soc., 142 (2014), pp. $581-590$.

[MMN] H. Markwig, T. Markwig, and A. J. Needergard, tropical.lib, a Singular 3-1-3 library for computations in tropical geometry, 2011.

[MY] J. MATHER, AND S. S.-T. YAU, Classification of isolated hypersurface singularities by their moduli algebras, Invent. Math., 69 (1982), pp. 243-251.

[Ma] H. Matsumura, Commutative Ring Theory, Cambridge Studies in Advanced Mathematics 8, Cambridge University Press, Cambridge, 1986.

[Mu] S. Mukai, An Introduction to Invariants and Moduli, Cambridge Studies in Advanced Mathematics 81, Cambridge University Press, Cambridge, 2003.

[O] P. Olver, Classical Invariant Theory, London Mathematical Society Student Texts 44, Cambridge University Press, Cambridge, 1999.

[Sa] K. SAIto, Einfach-elliptische Singularitäten, Invent. Math., 23 (1974), pp. 289-325.

[Sch] M. Schulze, A solvability criterion for the Lie algebra of derivations of a fat point, J. Algebra, 323 (2010), pp. 2916-2921.

[W] H. WeBer, Lehrbuch der Algebra, 2 Auflage, 2 Band, Vieweg, Braunschweig, 1899.

[Y1] S. S.-T. YAU, Solvable Lie algebras and generalized Cartan matrices arising from isolated singularities, Math. Z., 191 (1986), pp. 489-506.

[Y2] S. S.-T. YAU, Solvability of Lie algebras arising from isolated singularities and nonisolatedness of singularities defined by $\mathfrak{s l}(2, \mathbb{C})$ invariant polynomials, Amer. J. Math., 113 (1991), pp. 773-778. 
A. V. ISAEV 